\title{
Axé! Santé, maladie et soins dans les terreiros de Candomblé de Bahia
}

\section{Leny Trad, Clarice Mota et Marieta Reis}

Traducteur : Marie Orfinger

\section{OpenEdition \\ Journals}

Édition électronique

URL : https://journals.openedition.org/pa/1537

DOI : 10.4000/pa.1537

ISSN : 2273-0362

Éditeur

Université Lumière Lyon 2

Édition imprimée

Date de publication : 4 janvier 2021

Pagination : 64-84

ISSN : 1634-7706

Référence électronique

Leny Trad, Clarice Mota et Marieta Reis, "Axé! Santé, maladie et soins dans les terreiros de

Candomblé de Bahia », Parcours anthropologiques [En ligne], 16 | 2021, mis en ligne le 04 janvier 2021 consulté le 13 avril 2022. URL : http://journals.openedition.org/pa/1537 ; DOI : https://doi.org/ 10.4000/pa. 1537 


\section{Axé! Santé, maladie et soins dans les terreiros de Candomblé de Bahia}

\section{Leny Trad}

Institut de Santé Collective, Université Fédérale de Bahia

\section{Clarice Mota}

Institut de Santé Collective, Université Fédérale de Bahia

\section{Marieta Reis}

Institut de Philosophie et de Sciences Humaines,

Université Fédérale du Rio Grande du Sud

Traduit du portugais par

Marie Orfinger

\section{INTRODUCTION}

L'expérience religieuse selon les termes proposés par Rabelo (1993) comprend la manière dont ses symboles sont vécus et continuellement resignifiés, à travers des processus interactifs concrets entre individus et groupes. Certains symptômes ou troubles corporels, compris dans la logique de la médecine officielle comme des troubles du corps physique et/ou de l'esprit, sont des signes ou des manifestations de spiritualité pour les religions de matrices africaines (Serra, Pechine \& Pechine, 2010 : 166).

Dans le cas spécifique du candomblé, on cherche à travers les rituels «à rétablir l'unité perdue entre l'aiê, le monde physique, la terre et l'orun, le monde surnaturel des entités divines ou orixás » (Rabelo, 2002:8). Comme le souligne Fábio Lima (2015), tous les Orixás ${ }^{1}$ sont impliqués dans la santé, dans le corps et son fonctionnement, comme Oxóssi et Oxalá, qui sont liés à la tête, à la direction et à la pensée, comme Exu, lié au fonctionnement des entrailles, des organes et des systèmes physiologiques ou comme Ogum, qui est lié aux

\footnotetext{
1 Les Orixás sont des divinités vénérées dans les terreiros, qui sont les lieux de culte des religions de matrices africaines. Le préfixe ori signifie "tête » (terme d'origine yoruba), de sorte que les candomblécistes croient être guidés, soignés et influencés par l'énergie de leur orixá. Les orixás sont souvent associés à des éléments de la nature et une partie du processus d'initiation consiste à se familiariser avec tous les mythes et rituels liés à leur " orixá de tête ", celui qui gouverne la tête du pratiquant de candomblé. [Note de la traductrice : le choix a été fait de respecter la graphie originale portugaise, comme Roger Bastide l'a fait dans Le Candomblé de Bahia (Plon, Paris, 2000), en signalant que le X se lit $\mathrm{CH}$ pour la prononciation].
} 
pieds et aux déplacements. D'autre part, l'auteur souligne que, s'il est vrai que les Orixás régissent les parties du corps et que dans les terreiros de Candomblé on trouve une compréhension relative à chaque organe du corps, cette perspective ne fonctionne pas par la logique de fragmentation, mais "par l'aspect relationnel de ces organes en mouvement". La dimension relationnelle est essentielle à l'existence des filhas et filhos-de-santos²; elle est l'axé lui-même (Lima, 2015).

Le terme axé est utilisé pour désigner de multiples éléments dans un terreiro de candomblé: ses «adeptes, ses coutumes et, par extension, son mode de culte ou sa Nation »(Opipari 2009: 86). Il est intéressant de remarquer, en particulier, l'utilisation de l'axé en tant que principe de vie, une sorte d'énergie vitale dont tout ce qui existe dans la nature est doté. Cette énergie vitale doit être constamment renouvelée et renforcée, et il existe de nombreuses façons pour ce faire. Prières, rituels, fêtes, nourriture des orixás, paó ${ }^{3}$, offrandes ${ }^{4}$, ebós ${ }^{5}$ et une série d'autres pratiques qui seront analysées dans cet article, exercent cette fonction. L'axé relie les gens aux orixás, à la famille, aux éléments de la nature et à leurs ancêtres, connotant une appartenance, comme dans l'échange d'expression de "peuple de saint » par le "peuple de l'axé », et ébranler cette force implique menacer sa propre existence (Póvoas, 1999).

Il convient également de noter que les temples afro-religieux sont devenus des espaces d'inclusion, de conseil et d'accueil, en particulier pour les groupes marginalisés, étant donné qu'en général ils sont situés dans les quartiers les plus appauvris de Salvador. Il est à noter, cependant, que le candomblé est inclusif pour tous ceux qui sont « choisis » par l'orixá, indépendamment de la classe sociale ou de la couleur de peau. En plus de ceux qui sont initiés dans les maisons de candomblé, un grand nombre de personnes fréquentent les terreiros, apprécient les fêtes et assument parfois des liens sporadiques avec la mãe de santo ${ }^{6}$ ou le pai de santo de la maison de candomblé.

Différentes études montrent que les rituels et les relations interpersonnelles qui ont lieu dans les terreiros de candomblé fournissent des échanges affectifs,

2 Filhas et Filhos de santo sont les personnes initiées dans le candomblé, littéralement les filles et fils de saint. On parle de saint au Brésil pour se référer aux orixás, d'une part par le syncrétisme avec le catholicisme, et d'autre part pour éviter les persécutions de la police, puisque le candomblé était une religion hors-la-loi jusque dans les années 1950 [ndt] et souffre de persécutions jusqu'à nos jours.

3 Une séquence rythmée de clappements de mains utilisée dans certains rituels du candomblé comme une révérence au sacré.

${ }^{4}$ Dans le candomblé, on croit à un lien spirituel de réciprocité entre les orixás et les êtres humains, qui est 'alimenté' par des offrandes. Des offrandes spécifiques correspondent à chaque orixá, avec des aliments précis, qui sont offerts à l'orixá en signe de remerciement ou pour une demande de protection/soins, ou même pour maintenir vivant ce lien sacré.

${ }^{5}$ Le mot ebó est un terme d'origine yoruba utilisé dans le candomblé. Les ebós sont également des offrandes aux orixás, qui renforcent le lien et l'échange d'énergies entre les orixás et les humains.

6 La mãe de santo est littéralement la mère de saint et le pai de santo, le père de saint, désignent le leadership le plus important dans la hiérarchie d'un terreiro de candomblé [ndt]. 
un accueil, de la production de connaissances, la promotion de la santé et la prévention de maladies et d'affections (Marmo Da Silva, 2007). Le terreiro est aussi un territoire de renouvellement de traditions millénaires, principalement au travers des connaissances et des pratiques liées à l'utilisation de plantes médicinales.

Pour comprendre un système thérapeutique religieux tel que le candomblé, qui a sa propre logique et rationalité, il est nécessaire de comprendre sa cosmologie, son ethos et sa vision du monde. Le présent travail se concentre spécifiquement sur les sens et les pratiques liées au corps, à la santé, aux maladies et aux soins dans le candomblé. Les aspects liés à la répartition des tâches de soins au sein du terreiro seront également analysés, en passant par le processus de partage des connaissances ancestrales. Ces éléments révèlent, à leur tour, la configuration de la hiérarchie dans les terreiros.

Les données partagées ici sont issues des incursions ethnographiques dans quatre terreiros de candomblés à Salvador (Bahia, Brésil) ou dans la région métropolitaine. Ces terreiros diffèrent en termes de taille, de leadership (mãe ou pai de santo), de succession (biologique et religieuse), d'ascendance ou de « nation" ${ }^{7}$ » d'origine. Pendant deux ans, nous avons siuivi un terreiro de taille moyenne, de tradition Angola, dirigé par une ialorixá ${ }^{8}$, dont la ligne de succession est biologique ; un petit terreiro de tradition Ketu, dirigé par un babalorixá, dont la succession est assurée par des liens religieux, définis par le jeu des cauris ${ }^{9}$; et un terreiro de tradition Jeje, de grande taille, dirigé par un babalorixá, également de lignée religieuse.

Il est à noter que l'idée de nation, telle qu'elle est présente dans le candomblé, acquiert une signification essentiellement théologique, utilisée pour désigner « le modèle idéologique et rituel des terreiros de candomblé de Bahia », dont les fondateurs de différentes régions d'Afrique ont transmis «aux groupes qui ont formé la norme des rites et le corps doctrinal qui se sont transmis à travers les époques et le changement des époques » (Costa-Lima, 1977: 21). Bastide (2001), en mentionnant l'existence des nations dans le Candomblé, le fait également au sens culturel et religieux, désignant chacune

\footnotetext{
7 Le terme «nation" dans le candomblé se reporte à l'origine ethnique et linguistique des groupes qui formèrent le candomblé au Brésil. Selon Prandi (2001 : 16), « le mot nation, dans le candomblé, exprime une modalité de rite dans lequel, malgré les syncrétismes, les pertes et les adoptions qui eurent lieu au Brésil, et même en Afrique d'où venaient les noirs, un tronc linguistique et les éléments culturels d'une certaine ethnie ont prévalus ». Les groupes du tronc ethniques/linguistiques yoruba ont formé le candomblé de nation nagô/ketu, les candomblés de nation jéjé trouvent leur origine dans les groupes ethniques parlant la langue fon et se distinguent par l'utilisation du terme voduns pour les divinités, par rapport au terme 'orixá' présent dans les candomblés ketu/nagôs. Les terreiros de nation angola proviennent du tronc ethnique et linguistique banto/quicongo/quimbundo et utilisent le terme inquices pour désigner les divinités. D'autres termes diffèrent également d'une nation à l'autre.

${ }^{8}$ Le terme 'ialorixá' signifie mère de saint ou mãe de santo et 'babalorixá' signifie père de saint ou pai de santo.

9 Le jeu de divination réalisé avec les coquillages appelés cauris en français et buzios en portugais. [ndt]
} 
de ces différences dans le domaine du culte et des rituels. En fait, la distinction entre les nations est présente dans la façon de jouer les tambours, dans les musiques, dans le culte de divinités spécifiques, dans la langue utilisée pour ces cultes, dans la durée de l'initiation ou d'autres traits rituels présents dans les maisons de candomblé.

D'autre part, nos interlocuteurs ont relativisé les démarcations entre les nations de candomblé à Bahia : que ce soit du Jeje, de l'Angola, du Ketu, de l'Ijexá, de l'Infante-ashanti, car tout est orixá, tout est inquice, tout est vodun (runtó $^{10} \mathrm{du}$ terreiro).

\section{SIGNIFICATIONS DE LA SANTÉ, DE LA MALADIE ET DES SOINS}

Tout au long de l'histoire, la médecine officielle a opéré une rupture entre la maladie et le social, construisant une représentation de la maladie comme un événement individuel, susceptible d'être isolé et interprété comme une morbidité spécifique d'un individu en particulier (Laplantine, 1991). Le concept de santé de la communauté des terreiros est basé sur la notion de sujet intégral, dans lequel il existe un lien entre le corps, l'esprit et l'âme. Les pratiques rituelles et les relations affectives construites dans le terreiro luimême permettent l'accueil, d'abord effectué par le Pai de Santo, le conseil et le traitement de ses adeptes, en intégrant dans ces pratiques les dimensions qui vont au-delà du champ physique.

Pour le candomblé, la santé est synonyme d'harmonie. La maladie est un déséquilibre entre les nombreuses polarités qui composent le monde et le sujet. Contrairement à une conception manichéenne, dans le candomblé rien n'est essentiellement bon ni essentiellement mauvais. La recherche de l'équilibre des forces intérieures et extérieures est ce qui favorise la santé et la vie. Pour maintenir cet équilibre, les filhas et filhos de santos doivent pratiquer des comportements adéquats, harmoniser les forces et renforcer l'axé au travers de rituels, de prières, d'offrandes et d'autres pratiques qui les guident à cette fin (Rabelo, 1998). Pour reprendre les mots d'une ialorixá interviewée : « la vie, c'est ça, c'est être capable de prendre soin de la nature pour qu'elle prenne soin de moi [...] la racine du poivrier rose fait revenir l'homme d'une transe [...]. La vie est une prière.... C'est prier Oxalá, lui demander de prier Iaori pour qu'il nous donne l'équilibre, pour qu'il nous donne la paix ».

La nature, l'homme et les divinités doivent être en harmonie constante, parce que l'orum et l'aiyê ${ }^{11}$, à travers la relation entre la personne et l'orixá, sont en communication constante et, par conséquent, leurs actions et leurs comportements se reflètent dans leur totalité. Dans cette perspective

\footnotetext{
${ }^{10}$ Dans la tradition Jeje Savalu, le runtó est le responsable des atabaques et des cantiques des voduns.

${ }^{11}$ L'orum est le monde des divinités, le monde spirituel. L'aiyê est le monde matériel, le monde des humains.
} 
holistique, la déstructuration de l'une des parties ébranle l'ensemble. Cette idée d'harmonie renforce certains éléments déjà traités : "être en bonne santé, c'est avoir l'équilibre spirituel... si tu as l'équilibre spirituel, je pense que physiquement tu te porteras bien aussi » (Lara, iajibona $\tilde{a}^{12} \mathrm{du}$ terreiro).

Le concept de santé est directement associé au maintien de l'axé. Ignorer cette nécessité peut justifier, dans bien des cas, l'apparition de maladies. La force de l'axé ne peut être objectivée ni instrumentalisée, elle n'est pas séparée du sujet qui la préserve. L'énergie est personnalisée, «c'est une valeur qui circule et qui qualifie les personnes, les objets et les lieux dans un même plan, sans distinction entre la valeur objective et le sujet valorisant » (Sansi, 2009: 154). Prandi (1991) souligne que l'augmentation de l'axé, l'énergie qui représente la force vitale du candomblé, est complètement enracinée et dépendante du bonheur de l'adepte; c'est dans le monde extérieur à la religion que le filho de santo est heureux, riche, beau, heureux au travail et en amour. Toute satisfaction et bien-être acquis dans la vie sont directement proportionnels à l'axé. Par conséquent, pour que l'axé soit positif, il est nécessaire d'établir une relation d'échange de faveurs entre les entités sacrées (orixás, voduns, inquices et caboclos ${ }^{13}$ ) et le filho de santo, entre chacun des filhos de santo, et/ou des relations réciproques avec des personnes importantes dans la société en général, d'autres clients de la maison, etc.

Il est très courant chez les filhas et filhos de santos, de mentionner les faiblesses et les problèmes de santé qui impliquaient leur passé sans candomblé. Ces situations sont interprétées comme une négligence dans la conservation de l'axé, ayant pour conséquence son affaiblissement. En général, la vie est toujours divisée entre un avant et un après la religion. L'idée de réciprocité, d'échange, est très présente dans cette notion, "donner pour recevoir ».

Chaque fois que je fais quelque chose comme ça (donner de la nourriture aux orixás), je sens un renouveau, je vais dans le miroir et je me regarde. Deux jours, trois jours, puis je me regarde. Et j'ai des surprises, quand je me sentais parfois laide, avec le visage d'une personne qui souffrait. Et puis quand je fais quelque chose, quand je remercie, quand je... j'ai l'impression d'avoir mon visage régénéré, ma joie renouvelée... Je ne sais pas, c'est une chose inexplicable. (Iabassê $\hat{e}^{14}$ de terreiro)

\footnotetext{
12 Terme similaire à mãe pequena (littéralement "petite mère »). Il s'agit d'une position immédiatement inférieure à celle de la direction religieuse, qui représente également la personne responsable des soins de la maison et une autorité religieuse.

${ }^{13}$ Le terme caboclo désigne les entités religieuses d'origine amérindienne, auxquelles un culte est porté dans la plupart des terreiros de candomblé de Bahia.

14 Dans la hiérarchie de la famille de saint, la Iabassê est responsable de la préparation des nourritures sacrées utilisées dans les rituels. C'est une fonction d'une grande importance, compte tenu que l'aliment est une grande source $\mathrm{d}^{\prime}$ axé.
} 
Dans le candomblé, les maladies ne sont jamais traitées comme de simples troubles physiques, leurs causes ont toujours une dimension spirituelle (Rabelo, 1998). Néanmoins, elles sont généralement divisées en deux catégories : les «maladies corporelles» et les «maladies spirituelles». Cependant, cette division est subtile, elle ne comprend pas une liste et définit encore moins le lieu où elle se manifeste. Par exemple, un mal de gorge peut être lié à un motif spirituel ou il peut simplement avoir pour cause d'être resté exposé au vent frais de la nuit. Il convient de noter que cette division, bien qu'elle ait été utilisée dans des études portant sur la religion et la santé, est employée au sein du terreiro, y compris par les divinités. Sa fonction est généralement utilisée pour diagnostiquer la cause du déséquilibre et non pour localiser la maladie. Il est entendu que la déstabilisation de tout élément du corps-complexe implique la déstabilisation des autres. Les parties ne sont pas indépendantes de l'ensemble, et vice versa.

Les sujets, initiés ou non, communiquent avec le monde des entités sacrées pour mieux vivre. C'est l'échange constant qui construit la vie en société. C'est dans le monde terrestre que le bien-être est recherché et la force des divinités est indispensable pour atteindre le bonheur. Les explications et les interprétations sur les causes qui ont provoqué les afflictions et les maladies ne sont jamais enfermées dans des justifications physiologiques. Même les maladies identifiées comme maladies du corps impliquent des explications qui sont liées à l'identité sociale du sujet, à ses relations avec les autres et avec le monde. Elles relèvent par conséquent du domaine culturel.

Les sociétés noires africaines ne sont pas sans connaître les causes naturelles, matérielles et physiologiques des maladies. Mais leur recherche d'explication commence exactement là où l'explication scientifique se termine, situant les véritables causes des maladies dans le domaine humain et culturel, les causes scientifiques étant considérées seulement comme matérialisation et moyens choisis pour manifester la maladie. Il n'importe pas de poser la question «comment c'est arrivé », comme le ferait le scientifique, mais bien de s'interroger sur "pourquoi seulement cette personne et pas cette autre. » (Munanga, $2007: 14$ )

En plus de cette perte ou de cet affaiblissement de l'énergie vitale, qui laisse place à l'apparition de la maladie, il existe d'autres facteurs explicatifs de son apparition chez les individus. Nos relevés ethnographiques coïncident parfaitement avec la taxonomie proposée par Barros et Teixeira (2000), qui envisage quatre autres éléments qui prédominent dans le schéma causal complexe à propos du caractère surnaturel des maladies dans le candomblé. Les deux premiers désignent l'action ou la «marque » d'un orixá sur l'élu ou l'individu choisi pour accomplir l'initiation partielle ou totale, ainsi que sur un initié qui a négligé ses obligations religieuses ou sociales. Parmi les pères, les mères, les filhas et filhos de santos des quatre terreiros, on trouve beaucoup d'histoires qui se réfèrent à cet "appel », quelque chose qui se manifeste à travers un symptôme persistant, une configuration qui a été déterminante 
pour l'initiation au candomblé. Après tout, sans la feitura ou initiation, il n'y a pas de fin à la maladie dont on souffre.

En ce qui concerne le deuxième élément, nous devons rappeler que l'initiation signifie un engagement religieux et social. L'expression « obligation » elle-même met l'accent sur le sens du devoir à accomplir, ce qui inclut la participation aux fêtes, à la vie quotidienne du terreiro, aux offrandes, etc. Ces processus garantissent le renforcement des liens entre les filhas et filhos de santos et le sacré, et entre eux. Le terme ximba ou "raclée du saint » est utilisé pour exprimer les maladies qui résultent de l'absence ou du non-respect de ses obligations.

La question des ximbas, généralement elles arrivent quand... Vous savez ce que vous avez besoin de faire pour prendre soin de vous mais vous n'y accordez pas d'importance. «- ah... peu importe...», «Je le ferai... dans quelques jours, dans quelques années ». Et alors les orixás... en fait, c'est le moyen que les orixás possèdent pour vous expliquer, pour attirer votre attention sur cela, d'une manière plus légère ou plus dure. (Cida, ialorixá du terreiro)

Ensuite, il y a la violation des règles, la transgression des tabous alimentaires ou des interdictions sexuelles. La transgression, dans ce cas, ne se réfère pas seulement aux diktats sacrés, bien qu'ils soient nombreux sinon majoritaires dans les terreiros, puisque tout est défini par ordre des orixás. L'initié n'a pas respecté ses quizilas, c'est-à-dire les restrictions alimentaires, les tabous liés à l'orixá de tête et par conséquent, comme l'un des initiés l'a rapporté, « il y a une certaine chose que je ne peux pas manger car je me sens mal».

Et, enfin, il y a la contamination par contact avec les eguns. Il s'agit d'un type de causalité couramment identifié, surtout chez les personnes de l'extérieur du terreiro qui cherchent de l'aide. Les eguns sont des esprits qui « ont déjà fait le passage », c'est-à-dire des esprits de morts qui, comme une sorte d'envoûtement, causent des problèmes physiques ou psychologiques à ceux qui sont affligés par une telle situation (Reis, 2012) ${ }^{15}$.

Par conséquent, même si la cause d'une maladie n'est pas ignorée dans son contexte physiologique et naturel, la justification de son apparition est additionnée aux autres termes sociaux. Le "mauvais œil » et les énergies négatives en sont quelques unes. Elles affaiblissent les gens et permettent à la maladie de s'installer, laissant l'individu sensible à la maladie. Elles sont fondées sur des relations sociales qui présupposent l'envie, l'égoïsme et la vengeance, par exemple. Le mauvais œil peut constituer la maladie elle-même, c'est-à-dire affaiblir l'individu. Normalement, ce type de maladie est lié à des faiblesses comme la diarrhée, l'anémie, le malaise, la fatigue et l'indisposition.

\footnotetext{
15 Nous avons repris ici des éléments de la dissertation de Master de Marieta Reis, co-auteure de cet article, dont la recherche s'est concentrée sur l'un des terreiros mentionné.
} 
S'insérer dans le contexte religieux du candomblé signifie être protégé, selon la conception des fidèles. C'est une compréhension émique du corps, qui peut être plus ou moins vulnérable et sensible à la maladie selon le respect des obligations rituelles et de la culture de l'axé. Les membres des terreiros croient qu'il y a une influence sur la santé de leurs visiteurs en fonction de leur fréquence dans le terreiro et de l'accomplissement de leurs obligations. Selon nos informateurs, les fils de saints qui s'éloignent beaucoup du contexte religieux du candomblé sont plus susceptibles d'être malades.

La maladie peut également provenir de la réalisation de certains rituels de manière erronée, comme l'initiation par exemple. L'un de ces cas a conduit une femme à avoir beaucoup de problèmes de santé. Tout a commencé quand elle est arrivée au terreiro, déjà initiée comme equede ${ }^{16}$ de Oxóssi, quand elle a été avertie par Mère Carol que son initiation avait été faite erronément. Le jeu de cauris avait révélé qu'elle n'était pas de Oxóssi et qu'elle n'était pas non plus equede, mais bien rodante ${ }^{17}$. Une telle erreur peut mener à souffrir de maladies psychiques, et même entrainer la mort :

Comme je te l'ai dis, chaque cas est un cas. Il y a des gens qui ont de graves problèmes, il y a des gens qui vont jusqu'à perdre la vie, il y a des gens qui sont consternés, des gens qui tombent malades. Et il y a des gens, tu sais? Qui n'ont rien, apparemment il ne leur arrive rien, mais si on regarde de plus près, parfois la personne veut réaliser quelque chose et $n^{\prime} y$ arrive pas. Tu comprends ? C'est très... l'orixá, il agit méticuleusement. (Ogãa18 d'un terreiro)

Comme l'indique Reis (2012), la tête a une signification importante dans le candomblé. C'est en elle que se trouve le dieu individuel de chacun. Appelée Ori, elle est divisée en deux parties, la physique et la spirituelle. Le front, le cou, les côtés et le dessus sont remplis de significations. La tête est un lieu d'énergie et d'orixá, c'est à travers elle qu'ils viennent sur terre. Les gens de l'axé ne touchent généralement pas la tête des autres et n'aiment pas non plus que les autres touchent la leur.

Si la tête n'est pas équilibrée, le corps est également en déséquilibre. Parce que les gens de l'axé n'aiment pas... mettre leurs mains sur la tête... Mais c'est un endroit qui.... Quand l'enfant naît, ceci ici [montre le crâne] commence à palpiter. C'est donc un endroit... avec le temps, elle se fermera, d'accord, la fontanelle. Si vous

\footnotetext{
${ }^{16}$ Les equedes sont les femmes qui ne rentrent pas en transe et ont pour responsabilité de prendre soin des orixás.

17 Rodante est le terme utilisé pour classifier la personne qui est sensible aux incorporations de divinités, c'est à dire, qui reçoit des orixás, des caboclos ou d'autres divinités durant la transe rituelle.

18 En yoruba, le mot signifie «Seigneur de ma maison ». L'Ogã est un prêtre choisi par les orixás, il est de grande importance dans les rituels. C'est une charge exercée seulement par les hommes. Ils deviennent responsables des chants, des rythmes, des sacrifices des animaux, de la cueillette des feuilles, etc.
} 
avez de l'énergie négative et tout, ici [la tête] c'est un lieu d'énergie aussi, c'est l'endroit où on plante l'orixá, il y a tout ce problème... alors la personne vient, en sortant d'un enterrement, ou la personne est chargée et tout, et elle pose sa main sur votre tête là, vous pouvez immédiatement sentir un mal de tête. (Adanna, Iajibonã)

L'on perçoit plus clairement l'interrelation entre les «maladies corporelles » et celles qui sont «spirituelles » dans les maladies qui se manifestent dans d'autres parties du corps. Comme nous l'avons déjà dit, même les maladies reconnues pour des causes organiques ne sont pas exemptes d'une justification religieuse, spirituelle ou sociale, et ceci se reflète dans leur traitement. Munanga (2007) affirme que les relations des sujets avec les autres et avec le monde qui les entoure, ont une influence directe sur la santé, dans la mesure où une vision anthropocentrique du monde prédomine dans les cultures de matrice africaine (Reis, 2012).

Les données ethnographiques recueillies suggèrent que le concept de soin présent dans le terreiro est clairement proche de l'idée de veiller sur. De fait, l'association entre les notions de soigner et de veiller sur, est continuellement exprimée dans les terreiros, en commençant par la dénomination même du chef spirituel, connu sous le nom de soigneur ou veilleur (zelador).

\section{LES PRATIQUES DE SOINS : CORPS, NATURE, ÉQUILIBRE ET SPIRITUALITÉ}

L'axé s'accumule dans les fundamentos ${ }^{19}$ de la maison, dans les objets rituels, dans le terreiro lui-même et dans le corps des personnes qui s'unissent et partagent cette énergie. En général, tous les traitements effectués dans le terreiro passent par la mãe (ou le pai) de santo. Si, dans certains cas, ce n'est pas elle qui conduit le traitement, c'est elle qui le rend possible. Le diagnostic des maux est fait par elle à travers le jeu des cauris. Cependant, pour ses filhas et filhos de santos, le jeu de cauris n'est pas utilisé avec la même fréquence que pour ceux qui visitent le terreiro sporadiquement. Elle connaît l'odu ${ }^{20}$ de ses filles et fils et les orixás qui gouvernent leur destin et, généralement, la compréhension des problèmes lui est donnée à travers son interprétation des actes, des situations et des comportements. Que ce soit avec l'usage du jeu de coquillages ou non, la mãe ou le pai de santo exerce la fonction d'interpréter les faits qui conduisent aux attitudes à adopter (Reis, 2012).

19 Ce sont les caractéristiques de bases spirituelles (les fondements) du terreiro, parfois matérialisées en objets sacrés, parfois en mythes qui expliquent les qualités et prohibitions liées à chaque orixá [ndt].

${ }^{20}$ L'odu est une histoire mythologique associée aux différentes positions des coquillages, qui raconte le destin de la personne. Il est fortement lié à son orixá de tête et à la mythologie qui accompagne cet orixá, mais il assume également des qualités propres, liées à la personne. 
La famille du terreiro est responsable de l'accompagnement psychosocial, du soutien et des échanges à travers un réseau de relations affectives et de solidarité. Les moments d'écoute et de conseil sont présents tant dans la vie quotidienne des fils de la maison que pour ceux qui viennent chercher de l'aide. Même si les gens n'ont pas les ressources financières, un service, un mot et une alternative pour permettre la résolution ou l'inversion du problème sont toujours disponibles. Le soutien apporté par le terreiro, avec l'aide de ses membres, et la relation qu'il établit avec sa communauté, font du terreiro un dispositif qui fonctionne comme un réseau de soins de santé.

La notion de soins peut faire référence à diverses significations et pratiques. Dans les terreiros de candomblé, il y a des allusions récurrentes à "prendre soin de la tête ", " prendre soin du corps », " prendre soin de l'orixá ». Les discours obtenus lors des entrevues avec les adeptes du candomblé et les représentations religieuses du candomblé dans la société contemporaine montrent l'importance de la relation corps et esprit dans l'action et la réflexion sur la santé. Les adeptes de cette religion d'origine africaine croient que les individus ne sont pas dissociés du domaine spirituel, et qu'il existe un lien entre ces deux domaines: le matériel et le spirituel. La nature et les éléments de la nature sont importants dans le processus de guérison, parce qu'ils sont liés à l'équilibre de l'esprit avec le corps. Les plantes, les animaux, les minéraux, l'eau et d'autres éléments de la nature établissent l'équilibre nécessaire pour que l'homme soit dans le monde.

Certaines actions présentes dans les terreiros fonctionnent comme un sauvetage des traditions, qui gagnent un sens profond dans cet espace partagé par les croyances et les valeurs culturelles. Le respect des personnes âgées et de leur sagesse, le contact corporel, la célébration de la vie, de la naissance et de la mort, le respect des orientations sexuelles, l'harmonie psychosociale, l'inclusion de la diversité, etc. sont des exemples de pratiques qui contribuent aux soins de santé. Ainsi, les pratiques développées dans les terreiros de candomblés constituent des instruments importants pour promouvoir la citoyenneté, le respect, l'appréciation de l'autre et de soi-même, où les personnes peuvent parler de leurs pensées et sentiments, sans jugement moral de la société.

Les pratiques et les rituels de guérison peuvent varier selon les raisons qui ont causé la maladie, avec une séparation entre les causes spirituelles et physiques. Lorsque les symptômes des sujets indiquent des maladies reconnues comme biologiques, le soutien offert dans le terreiro consiste à conseiller et à guider vers une meilleure alternative de guérison. Dans ce sens, il peut y avoir une association entre les soins médicaux et les soins fournis dans le terreiro. Les fidèles eux-mêmes aident le pai de santo à distinguer et à orienter leurs symptômes à la recherche du traitement qui leur semble le plus efficace.

Les fidèles nous ont parlé des différentes pratiques utilisées dans le terreiro pour la restauration de la santé, telles que l'ebó, l'utilisation d'herbes 
médicinales, le bori21, les bains de feuilles, les bénédictions, les boissons, le nettoyage du corps et de l'esprit. Toutes ces stratégies sont décidées à travers le jeu des cauris, car la communication avec le monde spirituel est fondamentale pour choisir les pratiques et les conseils. Les stratégies de soins peuvent aussi être offertes sous forme d'offrandes/dons aux orixás, soit pour demander que quelque chose se produise, que quelque chose soit défait, ou en reconnaissance pour le désir réalisé.

L'aide des orixás délimite un système d'échange et de réciprocité entre les hommes et les divinités, dans lequel la religion joue le rôle de médiateur des malades pour atteindre la guérison. Ces moyens sont généralement liés à des sacrifices, des travaux et/ou des dons à l'orixá qui effectuera la guérison. Les orixás demandent des obligations et des offrandes aux êtres humains, en échange de la solution à des problèmes de santé ou de la transformation de l'expérience de la souffrance. En général, les offrandes impliquent le sacrifice d'animaux, choisis avec soin, en fonction de l'orixá spécifique (Prandi, 2001).

Parmi les pratiques rituelles qui contiennent des propriétés thérapeutiques, les plus courantes sont les purifications, les bains de feuilles, les ebós, le bori et l'initiation elle-même, si nécessaire. En plus de la connaissance acquise par une ialorixá ou un babalorixá dans sa trajectoire religieuse, s'ajoutent également les pouvoirs sacrés conférés par les orixás. Dans la vision d'une ialorixá, elle sert d'intermédiaire entre ceux qui cherchent de l'aide et les Orixás : "En fait, je suis, j'ai l'habitude de dire aux jeunes que je suis un grain de sable devant l'océan, je ne suis personne, je suis juste une boîte, je dis aux jeunes que je suis un appareil ».

La base fondamentale pour le traitement des problèmes dans le terreiro, ce sont les herbes, les plantes. Fondamentalement, toute la guérison dans le terreiro se fait à partir de ces herbes. Le Doté22 transmet un peu de ses connaissances et de ses savoirs à la personne qui consulte au terreiro, en lui apprenant à prendre soin d'elle-même à partir de ses enseignements avec les herbes, en faisant du thé, en plaçant certaines herbes sous l'oreiller, etc. "Seulement la plante, l'herbe est la base. Nous révérons les herbes et nous guérissons les gens avec les herbes » (extrait d'une interview donnée par Hamilton, pai de santo de terreiro).

Il n'est pas rare que les traitements religieux apparaissent côte à côte avec la biomédecine. En comparant les traitements religieux aux traitements biomédicaux, de nombreux chercheurs observent que les premiers investissent beaucoup plus dans la communication, réussissant à produire un accord intersubjectif qui fait souvent défaut dans les approches biomédicales

21 Le mot bori signifie « donner à manger à la tête » avec pour but de fortifier la tête, s'occuper de la santé et du bien-être, et de renforcer le lien avec l'orixá, avec la famille de saint et avec le terreiro lui-même.

22 Terme utilisé pour désigner le leader d'un terreiro Jejé, le poste équivalent à mãe de santo ou ialorixá 
(Comaroff, 1980). Les interfaces thérapeutiques que les sujets favorisent dans les soins de santé sont plus récurrentes lorsque les maux sont interprétés comme des "maladies du corps». "La santé physique est traitée par le médecin, n'est-ce pas ? Nous n'avons pas ce pouvoir, nous aidons juste les choses à avancer. Et la santé spirituelle est traitée par le pai ou la mãe de santo » (muzenza $a^{23}$ du terreiro).

Bien que les traitements soient basés sur des logiques et des rationalités clairement distinctes, ceux-ci sont considérés comme complémentaires et la combinaison des pratiques thérapeutiques du terreiro et de la médecine formelle est fréquente chez les adeptes (Silva, Dacach, Lopes, 2005 ; Ferreti, 2003). Alors que les premiers sont orientés à partir de leur cosmologie holistique et comprennent la santé à partir de la multiplicité d'éléments et d'événements qui sont interconnectés, permettant l'harmonie et l'équilibre, les seconds, par leur spécialisation et leur linéarité, ont tendance à déconnecter le social du biologique.

Nous avons relevé également une compréhension syncrétique de la causalité - une maladie d'origine physique et en même temps spirituelle -, ce qui nécessiterait un double traitement et l'espoir que la médecine formelle associée au candomblé aiderait, donnerait une réponse positive, en potentialisant les traitements. Le terreiro est sollicité pour augmenter les chances de résoudre les afflictions. Même si, en même temps, les adeptes utilisent le système médical formel pour se guérir de maladies physiques/psychologiques. «(...) En général le traitement, c'est en commun. La personne continue d'aller chez le médecin et va aussi offrir des ebós pour se fortifier, pour que le traitement médical fasse plus d'effet » (extrait d'une interview avec Rodrigo, filho de santo du terreiro). S'il s'agit d'une maladie du corps, le doté a tendance à guider le patient à rechercher une ressource médicale formelle, ne l'empêchant pas d'effectuer également un traitement spirituel dans le terreiro:

La maladie du médecin, seul le médecin peut la guérir, et notre maladie, la maladie qui nous appartient, il ne la guérira jamais. C'est la feuille qui la guérira. C'est un bain. C'est un secouement24, c'est un ebó qui va guérir, si tu ne le fais pas, ça ne va pas guérir (...) Quand les personnes arrivent, on va regarder, chercher conseil et si on voit que la maladie est médicale, on envoie la personne chez le médecin, car je ne suis pas un fanatique, je suis un religieux. (Allan, Pai de Santo de terreiro Jeje)

Si la maladie est liée à quelque chose avec egun 25 , ou Exu, ou tout autre, ou avec un orixá, quoi que ce soit, cela va avoir une influence. Si ce n'est pas le cas, si la

\footnotetext{
${ }^{23}$ Le terme muzenza désigne une personne débutante dans le candomblé d'origine angola, c'est le terme équivalent à iaô dans le candomblé ketu.

24 Le terme "secouement» (sacodimento) désigne un rituel de purification spirituelle, pour rétablir la santé et fortifier l'axé.

${ }^{25}$ Les esprits des ancêtres qui restent sur Terre.
} 
maladie n'a rien à voir avec ça, cela aidera à avoir une bonne énergie, à avoir une protection. Mais je n'accepterai jamais le Pai de Santo qui dit: "n'allez pas chez le médecin, non »... Vous êtes fou ? (...) le bon pai de santo essaiera de protéger de tous les côtés si la personne est malade, il essaiera de rechercher dans le jeu de cauris pourquoi, s'il y a un problème lié à la partie spirituelle, à la partie métaphysique. Mais la personne doit aussi aller à l'hôpital, doit aller chez le médecin, doit prendre soin de soi, tu comprends ? (Jânio, initié, terreiro Ketu)

Selon un ogã, au fur et à mesure que l'on se familiarise avec le candomblé, il est possible de développer une sensibilité pour distinguer la maladie spirituelle de la maladie physique. La personne n'a pas nécessairement besoin d'être initiée pour avoir cette sensibilité, il suffit qu'elle soit déjà impliquée dans ce contexte religieux pour ressentir et identifier, " parce que vous savez immédiatement, quand c'est une maladie normale, parce que vous sentez aussi que ce n'est pas un état normal, ça vous donne envie de pleurer, de vomir, de tout casser » (Julia, petite fille de la mãe de santo, pas encore initiée).

L'orixá de tête est révélé par le jeu des cauris, ainsi c'est tout un univers archétypal qui s'ouvre, conduisant le sujet à découvrir des analogies entre les récits mythiques des orixás et sa propre vie. En ce sens, «la vie des individus et tous les événements qui peuvent s'y produire, reflètent une mythologie et une cosmologie assez complexe » (Mota et Trad, 2011: 332), interférant également dans la réinterprétation des épisodes de maladie : "Je suis d'Omolu, donc, j'ai cette certitude en moi : c'est comme ça! Tu sais ?....Quand j'étais enfant, j'avais des problèmes de peau, des éruptions de chaleur, toutes ces choses, qui sont liées à Omolu » (visiteur d'un terreiro Ketu).

Le soin pour garder le corps en bonne santé, pour cultiver l'axé, pour se protéger des influences extérieures négatives se fait par la purification, les bains de feuilles, les secouements, l'utilisation de colliers de perles : «ce que nous utilisons est le collier, les perles... et toujours faire la purification $\mathrm{du}$ corps » (initié dans le terreiro Ketu). Mais surtout, l'entretien continu du lien avec les forces spirituelles, avec les orixás, est indispensable. Il est à noter que le candomblé possède son propre système de classification des maladies, ainsi qu'un sens du corps, basés sur la relation avec l'orixá (Mandarino et Gomberg, 2013). Dans ce processus, les récits mythiques de l'orixá qui gouverne la tête du filho de santo et les éléments nécessaires à sa vénération de l'orixá (les aliments préférés de chaque orixá, son rythme, sa danse, etc.) sont en dialogue: si le corps est pensé et vécu comme l' «habitat de l'orixá», la santé et l'équilibre en dépendront (idem : 209).

\section{LA FAMILLE DE SAINT, L'AUTORITÉ ET LA TRANSMISSION DU SAVOIR DANS LE TERREIRO}

$\mathrm{Au}$ cours des siècles de pratique esclavagiste, d'innombrables familles africaines ont été désintégrées du fait de la traite des africains. Non seulement 
les liens biologiques de parenté ont été rompus, mais les groupes ethniques ont été séparés, rompant avec les anciennes formes d'organisation socioculturelle. Cependant, malgré la difficulté de maintenir les relations familiales et d'accomplir les rituels, les Africains emmenés au Brésil ont trouvé des formes parallèles d'organisation sociale dans la religiosité. Ainsi, la rupture avec les anciennes formes d'organisation socioculturelle a donné naissance à la famille de saint comme réélaboration de la configuration familiale à l'intérieur du terreiro (Costa Lima, 2003).

Selon Prandi (2004), la famille de saint est le résultat de la reproduction de la structure de la famille polygénique africaine, qui prescrit des modèles d'inceste parmi les frères et sœurs de saints et une structure hiérarchique avec des positions et fonctions délimitées.

La mãe de santo ou le pai, a toujours été l'autorité suprême du terreiro et toutes les décisions qui, selon la croyance du candomblé, expriment la volonté de l'orixá propriétaire du terreiro, qui est la même que celle de la mãe ou pai de santo, sont indiscutables. La mãe de santo est la mère de la famille spirituelle, la famille-desaint et elle est la propriétaire de fait du lieu de culte, et bien que l'ensemble du groupe soit structuré en hiérarchies et positions qui dépendent du temps d'initiation, en relations de parenté et en fonction des obligations initiatiques déjà remplies, la désignation des fils-de-saint à des postes prestigieux et la nomination à des fonctions rituelles impliquant le partage du pouvoir de la mère dépend uniquement et exclusivement de la volonté de la mãe de santo (Prandi, $2004: 4$ ).

Quand on parle de la famille de saint, on peut l'identifier au concept d'institution sociale familiale, car les liens créés dans le candomblé par le processus initiatique sont effectivement familiaux (Costa Lima, 2003). Comme toute structure familiale, les différences de genre interfèrent dans les relations et, dans le cas du candomblé, le profil des leaders et la dynamique du terreiro ont une influence. Selon certains informateurs, il n'y a pas de différence entre le pai et la mãe de santo quant à leur rôle de gardiens de la maison et de leurs enfants, ni quant à la disposition verticale des rôles joués dans l'espace religieux. Cependant, certains interlocuteurs ont souligné la différence dans la manière de traiter les fils et filles de saint. Un og ã a montré sa préférence : « une femme pensera d'une certaine façon, un homme d'une autre [...]. Je préfère, en particulier, même lorsqu'on me le demande, que la personne cherche une mãe de santo pour être sa gardienne. » Nous avons entendu sur le terrain que le pai de santo exerce généralement son rôle d'une manière plus « dominatrice », reproduisant son rôle et occupant la place la plus élevée de la hiérarchie du terreiro d'une manière plus autoritaire. La mãe de santo semble représenter l'extension de la mère biologique, dotée de condescendance, de compassion et de sentiment maternel, qui prend soin de ses enfants avec amour et affection.

Il est à noter que les relations de respect et d'obéissance se sont révélées similaires dans les quatre terreiros, quel que soit le sexe du chef de culte. Il y a 
un grand respect dans tous les terreiros pour le pai ou la mãe de santo, qui concentrent les rôles de leadership, d'administration de la maison et des décisions rituelles. L'admiration qu'ils suscitent parmi les participants de la communauté du terreiro est d'une grande importance pour l'organisation de sa structure sociale, car elle crée des liens forts de dépendance émotionnelle et psychologique avec lui et avec l'environnement dans lequel ils sont insérés. Quand quelqu'un arrive dans le terreiro demandant des soins spirituels pour une souffrance quelle qu'elle soit, c'est à de rares exceptions près la ou le chef de culte qui peut examiner cette personne et qui, à travers le jeu des cauris, découvre quel processus l'individu doit suivre pour résoudre ses problèmes. Dès lors, la ialorixá ou le babalorixá délibère et distribue les tâches que chaque fils de saint réalisera en fonction des permissions que chacun possède pour exercer de telles fonctions.

Même absent, en voyage, par exemple, elle/il exerce un certain contrôle sur le comportement des fils de saint. En cas de maladie de la ou du chef de culte, toute la dynamique du terreiro est changée, et c'est une situation redoutée par tous. C'est pourquoi le soin porté à sa santé est extrêmement important, comme l'expriment les récits suivants de fils de saint, recueillis dans deux terreiros : "s'il tombe malade, on tombe malade, c'est impossible à éviter! Nous devons prendre soin de lui parce que c'est lui qui a le savoir à nous transmettre »; « si le problème est spirituel, il peut en effet influencer les fils de saint. Parce qu'elle est la matriarche, elle est la base, donc si la base est ébranlée, toute la structure sera compromise... Si Mère Carol tombe malade, tout le terreiro tombe malade avec elle ».

En fait, lorsque la ialorixá ou le babalorixá tombent malades et ne peuvent pas faire leur travail habituel, le rythme du terreiro change, la vie quotidienne est fortement affectée. Par exemple, les dates des fêtes commémoratives sont modifiées. Une situation a été citée, dans laquelle le Doté d'un des terreiros étudiés a subi un accident vasculaire cérébral. Sa souffrance face à la maladie a été partagée par l'ensemble du collectif dans le terreiro, dont la dynamique et le fonctionnement ont été profondément affectés par cet événement. Dans ces situations, on peut se tourner vers le pai pequeno ou mãe pequena ${ }^{26}$, qui assume alors le commandement de la maisonnée.

Le degré d'engagement dans la famille de saint, ajouté au principe de l'ancienneté et du «temps d'initiation», sont des facteurs importants qui influenceront la dynamique hiérarchique de la maison. Les relations de pouvoir sont présentes dans les pratiques thérapeutiques impliquant la maladie et la guérison dans les religions ou les services de santé formels (Rabelo, 1993). Il en est de même dans les terreiros où la configuration hiérarchique et le rôle que chacun occupe dans cette hiérarchie au quotidien sont connus par la façon dont on se salue, par l'utilisation de colliers de perles,

\footnotetext{
${ }^{26}$ Op.cit. note 12 .
} 
par la position dans le xirê27 et même par le lieu où l'on s'assoie dans l'espace religieux (Simões, 2006). Certains aspects sont cruciaux pour distinguer les relations : l'ancienneté, c'est-à-dire " l'âge de saint "; la connaissance rituelle des fondements de la religion, transmis oralement; les manières de saluer les différentes positions hiérarchiques; le comportement de soumission et d'autorité des personnes dans leur position; l'utilisation d'instruments évocateurs par l'autorité pour contrôler les orixás des les fils de saint de la maison; les sièges du salon des fêtes disponibles seulement aux autorités et dirigeants; les accessoires et les vêtements utilisés comme signes d'appartenance à un certain orixá, et comme distinction entre les membres de la hiérarchie.

C'est une question de réciprocité : plus le sujet est présent, plus il remplit ses obligations et se donne, plus il reçoit respect et considération, et ainsi les liens sociaux et affectifs tendent à s'approfondir. Plus cette présence se raréfie, plus la distance par rapport à ce noyau augmente. Les personnes qui ne font pas partie du noyau familial peuvent être distinguées en "clients" et «parents de saint ${ }^{28}$. Le premier groupe se caractérise par des liens plus superficiels et moins intenses, et beaucoup sont des amis ou des parents des "gens de la maison ». Sur le plan matériel, la maison est maintenue par la contribution de ces clients. Les filhos de santos contribuent aussi financièrement, mais pas toujours de la même manière ou pas assez d'un point de vue économique. Comme l'a dit l'un des membres de cette famille de saint: «Celui qui donne le plus d'argent au candomblé n'est pas le filho de santo, c'est le client ». La relation est donc plus complexe qu'il n'y paraît.

La condition de client est considérée comme une phase préalable à l'immersion complète dans la religion. Ces visiteurs sont d'une grande importance pour légitimer l'efficacité et le pouvoir d'un terreiro et sa durabilité. Ils contribuent à soutenir matériellement la vie et les fêtes de la maison. Dans certains terreiros, un «travail » ou un ebó n'est pas payant du fait qu'il s'agit d'un service spirituel. Dans d'autres, les travaux doivent être payés pour que l'énergie puisse circuler, comme un échange, une sorte de réciprocité. Le paiement n'est pas toujours effectué uniquement avec une valeur monétaire et peut varier en fonction de la situation économique du client. L'échange de biens dans le réseau de candomblé donne sens à un circuit de permutations de services symboliques qui instituent des règles de coexistence et d'étiquette (Baptista, 2009).

Le second groupe, que nous avons désigné par l'expression «parents de saint », est constitué de personnes qui font partie du réseau de relations de la

\footnotetext{
27 Le xirê est la ronde où dansent les filhas et filhos de santos pour révérer et recevoir les orixás dans les fêtes publiques et privées des terreiros.

28 C'est un terme qui survint durant l'analyse des données, pour caractériser ceux qui fréquentent le terreiro, mais ne sont pas encore "faits» (initiés). Ils ne sont pas aussi proches que les fils de saint, mais ils font partie du réseau de relations de la maison, au même titre que des membres de la famille dans le cas d'une famille biologique.
} 
maison, mais qui n'ont pas encore accompli tous les rituels de l'initiation et qui n'auront peut-être jamais à l'accomplir. Certaines de ces personnes, en fonction de leur proximité avec la famille de saint, participent souvent aux fêtes de la maison et entretiennent le lien avec le terreiro par des bains, des purifications et des consultations périodiques. Dans les terreiros de la nation Ketu, on leur donne le titre d'abi $\tilde{a}^{29}$.

Il est nécessaire de souligner que les clients et les "parents de saint» peuvent, pour diverses raisons, devenir enfants de la maison. C'est ainsi que se configure la dynamique du terreiro, composée de ceux qui se trouvent à l'intérieur, de ceux qui se trouvent à l'extérieur et de ceux qui se trouvent au milieu du chemin, avec plus ou moins de proximité de la maison. Cette proximité peut être marquée par les rituels de l'obi ${ }^{30}$, du bori ou du lavage des colliers de perles (Costa Lima, 2003). Ce sont des rituels qui rapprochent aussi la personne de la dimension spirituelle, renforçant les liens avec son orixá régent.

L'expérience d'appartenance à un terreiro en particulier comprend l'apprentissage des formes traditionnelles, dans cette communauté, de comment prendre soin de soi, prendre soin du «saint» et prendre soin des autres, et sont transmises au sein de la famille de saint, principalement comme connaissance orale et par l'expérience et l'observation, dans et hors des rituels. Suivant la logique des religions d'origine africaine, la parole est soigneusement conservée : poser une question est une violation de la règle $d u$ silence et du respect, car on croit que la connaissance doit être transmise selon les mérites de chacun et en fonction du moment de l'initiation (Silva, 2006 : 44). Dans cet environnement, on apprend en observant, sans poser de questions ni faire preuve d'une curiosité excessive.

L'échange de présents et le don entre les personnes sont des pratiques centrales pour la compréhension de la vie de l'axé. L'axé, pour Sansi (2009), est le filho de santo lui-même, et ne peut être pensé en termes de capital symbolique, puisque la connaissance rituelle de l'initiation ne suffit pas à reproduire un axé d'une mãe de santo. D'autre part, il est nécessaire de considérer la possibilité qu'il y ait des échecs au cours de la vie. Les conflits et les contradictions, tels que les comportements qui dénotent l'avarice, la perversion ou la jalousie des mères ou des filles de saint, peuvent faire mourir une maison de candomblé et faire disparaître la connaissance rituelle ; en contrepartie, le « don » apparaît comme un moyen de contacter directement le « saint » et de renouveler le candomblé (idem : 154).

\footnotetext{
29 Terme en Yorubá qui signifie « celui qui va naître ». Abiã est un mot utilisé pour identifier une personne qui n'est pas encore passée par le processus d'initiation à la religion, mais qui a déjà été insérée par d'autres rituels.

30 Un type de noix blanche présente dans de nombreuses offrandes, qui se divise en quatre et qui mythologiquement contient les secrets du candomblé.
} 


\section{CONSIDÉRATIONS FINALES}

Dans le terreiro, des pratiques thérapeutiques sont produites à partir d'actions de prévention et de promotion de la santé basées sur une conception qui intègre le monde matériel et le monde spirituel. Selon les adeptes du candomblé, le monde matériel est visible et palpable, et le monde spirituel invisible et immatériel. C'est une manière d'être dans le monde et de comprendre les choses du monde lui-même, de manière différenciée, dans une logique qui intègre les dimensions mystiques et religieuses.

En ce sens, non seulement le corps est perçu comme quelque chose de dynamique et en construction permanente, mais, dans le candomblé, la notion même de " personne » a des spécificités. La personne se forme au fil du temps, dans la relation avec les orixás, avec la famille de saint et avec le terreiro luimême, une relation qui devient plus forte au fur et à mesure que l'axé, comme énergie vitale, se renforce en elle. Pour cela, tous les rituels sont nécessaires et constituent un système complexe de recherche de santé, de bien-être et de vitalité.

En réalité cet individu naît peu à peu, et d'une manière fort lente, puisque ce n'est qu'après vingt et un ans d'initiation que sa « personne » peut être complète, c'est-àdire que toutes ses composantes ont été individualisées, et donc elle aussi. Jusqu'à ce moment idéal, l'équilibre de votre moi est d'un type instable, très instable, dépendant de l'accomplissement de toute une série d'obligations et d'interdictions rituelles dont la violation, en détruisant cet équilibre, peut aller jusqu'à vous détruire en tant que personne, c'est-à-dire vous annihiler (Goldman, 1985 : 39).

Il y a un engagement symbolique et sacré envers les soins et un engagement historique envers la réciprocité.

Un autre aspect concerne la cosmologie de la religion. Les personnes et leurs relations avec le monde sont placées au centre des explications qui conduisent aux causes de la souffrance et doivent donc nécessairement être impliquées dans le traitement. Elles ne sont pas considérées comme de simples lieux de manifestation de la maladie, mais plutôt comme des sujets de celle-ci. Les actions menées sont basées sur la négociation, sur l'échange de symboles entre le sujet malade et l'agent de guérison, et sur l'implication de ce dernier dans les thérapies engendrées. La centralité que la maladie occupe dans la médecine scientifique est remplacée dans le candomblé par la centralité du sujet dans les traitements réalisés.

Les soins de santé ne comprennent pas seulement les soins personnels, mais surtout les soins de l'autre. On peut dire que la perception du corps et les modes de soins appris dans l'univers du candomblé sont en accord avec les conceptions d'intégralité et de soins humanisés présentes dans les propositions de modèles alternatifs de soins de santé. En particulier si l'on comprend l'intégralité des soins dans les termes de Gomes et Pinheiro, «comme une action résultant de l'interaction démocratique entre les acteurs, 
dans la routine quotidienne de leurs pratiques dans la prestation des soins de santé, aux différents niveaux de soins du système » (2005: 289).

Nous rejoignons Lima (2015: 30) quand il attire l'attention sur la nécessité de ne pas réduire la relation des fils de saints avec leur corps à la dimension culturelle et mythique, limitées à l'espace du terreiro. Dans le candomblé, on ne conçoit pas "l'idée du corps détaché de l'âme et de ses doubles", orixás, caboclos, etc. (idem). Il faut garder à l'esprit la communication « entre le système des représentations symboliques et le corps biologique lui-même » (ibid.), de sorte que faire partie du candomblé implique l'apprentissage de nouvelles techniques corporelles qui commencent à s'intégrer à la vie, à l'intérieur ou à l'extérieur du terreiro. C'est un processus d'apprentissage dans lequel « le filho de santo commence à prendre soin de son corps, avec la nouvelle emprise sur le monde qui est générée dans les rituels initiatiques» (ibid.).

Nous pouvons conclure que le candomblé est constitué comme un espace de soins, dans lequel les croyances et les pratiques sont partagées entre les sujets en faveur de la manutention de l'axé, aussi bien dans la vie des fils de saint que dans le terreiro lui-même. Dans la vie religieuse de matrice africaine, la réciprocité et l'échange entre fils de saints, visiteurs, parents, amis, voisins et dirigeants deviennent des phénomènes indispensables dans la configuration de cette toile qui est dynamique, car l'axé est toujours en mouvement.

\section{BIBLIOGRAPHIE}

Magali da Silva ALMEIDA, Pobreza, religião e cidadania: a tradição do candomblé e a luta pela cidadania na Baixada Fluminense. UERJ, 2009, [En ligne]. URL : http:// bibliotecavirtual.clacso.org.ar/ar/libros/aladaa/silva1.rtf

José Renato BAPTISTA, “Amigos amigos, negócio à parte... Mas, nem tanto assim: uma abordagem preliminar sobre as relações entre clientela e saúde no candomblé", in Ana Cristina MANDARINO et Estélio GOMBERG (orgs.), Leituras Afro-Brasileiras: territórios, religiosidades e saúdes, São Cristovão, UFS/EDUFBA, 2009, pp. 241-260.

José Flávio BARROS et Maria Lima TEIXEIRA, “O código do corpo: inscrições e marcas dos orixás", in Carlos Eugênio Marcondes de MOURA (org.), Candomblé: religião do corpo e da alma. Tipos psicológicos nas religiões afro-brasileiras, Rio de Janeiro, Editora Pallas, 2000, pp. 103-138.

Jean COMAROFF, "Healing and the cultural order: the case of the barolong boo ratshidi", American Ethnologist, v.7, n.4, 1980, pp.637-57.

Vivaldo da COSTA LIMA, A família de santo nos candomblés jeje-nagôs da Bahia: um estudo das relações intra-grupais, 2. ed., Salvador, Corrupio, 2003.

Vivaldo da COSTA LIMA, "O conceito de 'nação' nos Candomblés da Bahia", AfroAsia, 12, 1976, pp.65-90.

Mundicarmo FERRETTI, "Cura e pajelança em terreiros do Maranhão (Brasil)". I Quaderni del CREAM, Milano, v. 8, 2008, pp. 67-91. 
Márcio GOLDMAN, "A construção ritual da pessoa: a possessão no candomblé", Religião e Sociedade, Rio de Janeiro, Campus, 1985, pp.22-54.

Fábio LIMA, “Corpo e ancestralidade”, Repertório, Salvador, no 24, 2015, pp.19-32.

Ana Cristina MANDARINO et Estélio GOMBERG, "Candomblé, corpos e poderes". Perspectivas, São Paulo, v. 43, 2013, pp. 199-217.

Clarice MOTA et Leny TRAD, “A gente vive pra cuidar da população: estratégias de cuidado e sentidos para a saúde, doença e cura em terreiros de candomblé", Saúde e Sociedade, São Paulo, v. 20, n. 2, 2011, pp.325-337.

Reginaldo PRANDI, “O Brasil com axé: candomblé e umbanda no mercado religioso", Estudos Avançados, vol.18, n.52, 2004, pp.223-238.

Reginaldo PRANDI, "Candomblé e o tempo: concepções de tempo, saber e autoridade da África para as religiões afro-brasileiras", Revista Brasileira de Ciências Sociais, v. 16, n. 47, 2001, pp. 43-58.

Reginaldo PRANDI, Mitologia dos Orixás, São Paulo, Companhia das Letras, 2001.

Reginaldo PRANDI, Os Candomblés de São Paulo: a velha magia na metrópole nova, São Paulo, HUCITEC, 1991.

Míriam RABELO, "Entre a casa e a roça: trajetórias de socialização no candomblé", Religião e Sociedade, Rio de Janeiro, vol. 28, n. 1, 2008, pp. 176-205.

Marieta REIS, Do moço do anel às coisas do azeite: um estudo sobre as práticas terapêuticas no candomblé, Master en Santé Collective, Instituto de Saúde Coletiva, UFBA, Salvador, 2012.

Luis Felipe RIOS, Cinthia OLIVEIRA, Jonathan GARCIA et Richard PARKER, “Axé, práticas corporais e Aids nas religiões africanistas do Recife, Brasil", Ciência e Saúde Coletiva, v. 18, n. 12, 2013, pp. 3653-3662.

Roger SANSI, "Fazer o santo: dom, iniciação e historicidade nas religiões afrobrasileiras", Análise Social, vol. XLIV, n.1, 2009, pp. 139-160.

Ordep SERRA, Maria Cristina PECHINE et Serge PECHINE, "Candomblé e políticas públicas de saúde em Salvador, Bahia", Mediações, v.15, n.1, 2010, pp. 163-178. 
RÉSUMÉ : Cet article analyse les significations et pratiques sur le corps, la santé, la maladie et le soin, ainsi que les hiérarchies et dynamiques de transmission de connaissances ancestrales dans quatre maisons de culte (terreiros) de candomblé localisées dans la ville de Salvador et ses environs (Bahia, Brésil). Les terreiros se différencient de par leur taille, le profil des chefs de culte (hommes ou femmes), la forme de succession et d'ancestralité ou «nation» (Angola, Ketu, Jeje). Les incursions ethnographiques nous ont permis d'approcher des cosmologies pour lesquelles les causes des souffrances doivent être cherchées à l'intérieur même des personnes et dans leurs relations avec le monde et la nature. De ce fait, les pratiques thérapeutiques impliquent une relation d'interaction et de négociation entre sujets malades et agents de guérison. Pour le candomblé, le bien-être ou la santé s'atteint par la rénovation permanente de l'axé, principe de vie ou énergie vitale qui relie les personnes aux orixás, à la famille, à la nature, aux ancêtres, et dénote également une appartenance. Les prières, les rituels, les fêtes, les nourritures sacrées, les offrandes, entre autres pratiques observées dans les terreiros, visent à cultiver et renouveler l'axé.

MOTS-CLÉS : Significations, corps, santé-maladie-soin, Candomblé, axé, rituels, Bahia-Brésil 